\title{
SISTEMA DE GESTIÓN DE
}

\section{MANAGEMENT INFORMATION SYSTEM IN THE WAREHOUSES OF THE CONSTRUCTOR COMPANY OF ARCHITECTURE WORKS AND INDUSTRIAL \#4 OF GRANMA}

\author{
Ángel Enrique Figueredo León ${ }^{1}$ \\ Agustín Alejandro Ortiz Díaz ${ }^{2}$ \\ Elena Martínez Pérez ${ }^{3}$
}

1. Master en Informática Aplicada. Profesor Asistente de la Universidad de Granma, Bayamo, (Cuba). afigueredol@udg.co.cu

2. Doctor en Ciencias. Profesor Asistente de la universidad de Granma, Bayamo, (Cuba). aortizd@udg.co.cu

3. Ingeniería en Ciencias Informáticas. Profesora Instructora de la Universidad de Granma, Bayamo, (Cuba). emartinezp@udg.co.cu

Citación sugerida:

Figueredo León, Á.E, Ortiz Díaz, A.A. y Martínez Pérez, E. (2017). Sistema de gestión de la información en los almacenes de la empresa constructora de obras de arquitectura e industriales \#4 de Granma. $3 C$ TIC: Cuadernos de desarrollo aplicados a las TIC, 6(2), 28-37. DOI: $<$ http://dx.doi.org/10.17993/3ctic.2017.56.28-37/>. 
En la Empresa Constructora de Obras de Arquitectura e Industriales \# 4 de Granma en Cuba se realizan varios procesos dentro de los que se incluye la gestión de la información en los almacenes, el cual se torna difícil debido a que se efectúan mediante modelos confeccionados en Microsoft Office Excel y documentos impresos propiciando demora en la obtención de los resultados y la divulgación de la información. Atendiendo a estas necesidades la presente investigación tiene como objetivo desarrollar una aplicación web. Para su implementación, se empleó el framework Codelgniter en la programación de páginas dinámicas, PHP en su versión 5.5.11 como lenguaje del lado del servidor, HTML5 como lenguaje del lado del cliente, con soporte de base de datos en MySQL y la metodología de desarrollo Extreme Programing software.

\section{ABSTRACT}

In the Company Manufacturer of Works of Architecture and Industrial of Tree they are carried out several processes inside those that the administration of the information is included in the warehouses of the Company Manufacturer of Works of Architecture and Industrial of Tree, the one which you difficult restitution because they are made by means of models made in Microsoft Office Excel and printed documents propitiating delay in the obtaining of the results and the popularization of the information; what conditions the responsible personnel's exhaustion and the bad use of the available resources. Attending to this needs, this research aims to develop a web application. For its implementation, the Codelgniter framework was used in the programming of dynamic pages, PHP in its version 5.5.11 as server side language, HTML5 as client side language, with MySQL database support and development methodology Extreme Programing software.

\section{PALABRAS CLAVE}

Aplicación web, productos en los almacenes, gestión de información.

\section{KEYWORDS}

Web Application, Products in the warehouses, Information Management. 


\section{INTRODUCCIÓN}

Hoy en día, debido al auge en las nuevas tecnologías se trabaja en la digitalización y automatización de la información en todas las ramas de la sociedad, en la industria, las comunicaciones, el gobierno, la salud, la educación, entre otros sectores. El desarrollo de la Informática y las Comunicaciones ha traído como consecuencia que el intercambio de información entre las empresas, organizaciones y entidades sea más rápida, segura y eficaz.

Nada ni nadie escapan al paradigma y la nueva sociedad que ha creado las telecomunicaciones, la informática e Internet, y el mundo de la construcción no es ninguna excepción. Cada vez son más las empresas constructoras que se involucran en gestionar sus principales procesos de diseño, construcción e ingeniería empleando las tecnologías de la información y la comunicación para cumplir con calidad su encargo social (Ferrada y Serpell, 2009).

El Ministerio de la Construcción es el organismo rector de la política de desarrollo de los Servicios de Diseño, Ingeniería y Construcción, Producción de Materiales de Construcción y del Sistema de la Vivienda en Cuba, además de dirigir y ejecutar en lo que le compete y controla la política del Estado y el Gobierno en cuanto a las investigaciones ingeniero-geológicas aplicadas a la construcción (MICONS, 2014). Todo esto no podría ser posible sin la correcta gestión de los materiales de la construcción necesarios para los procesos antes mencionados, es por ello que es de vital importancia el trabajo que se realiza en los almacenes de las instituciones del MICONS. Dicho proceso genera información de gran utilidad para el personal que allí labora. Esta se lleva de forma manual mediante modelos impresos y documentos manuscritos, condicionando que en el aspecto informativo se presente una excesiva centralización de la información y un flujo abundante de documentos impresos. Adicionalmente, las personas que necesitan la información no disponen de ella en el momento y espacio adecuado. Esta situación genera problemas organizativos y no cubre todas las demandas informativas al incurrirse en atrasos en la entrega de reportes.

La Empresa Constructora de Obras de Arquitectura e Industriales \# 4 (ECOAI4) de Granma es una de las dependencias del Ministerio de la Construcción que dentro de su estructura presenta el proceso antes mencionado. Cuenta con una alta productividad y eficiencia económica logrando construcciones competitivas, dotado de un personal motivado y elevado sentido de pertenencia. También cuenta con toda la industria modernizada y máxima calidad en las obras minimizando los daños en el entorno con una fuerte imagen corporativa que permite satisfacer a los clientes nacionales e internacionales.

La gestión de la información es el proceso de organizar la información, evaluar, presentar, comparar los datos en un determinado contexto, controlar la calidad, veracidad, que sea oportuna, significativa, exacta, útil y que esté disponible en el momento que se le necesite (Vidal y Araña 2012; Torres 2015). Teniendo en cuenta esta consideración y en aras de solucionar la ineficiencia en el proceso de gestión de la información relacionada con los productos en los almacenes en la ECOAI4, se propone en esta investigación desarrollar una aplicación web que lo realice de manera rápida y eficiencia. 
Inicialmente, se hizo un estudio en la ECOAI4 para constatar las deficiencias en la gestión de la información relacionada con los productos en los almacenes y determinar la mejor vía para solucionarlo. Se utilizaron métodos y técnicas como el análisis y síntesis para recopilar y procesar la información necesaria, la revisión documental para conocer con claridad los datos que son de interés, la entrevista para obtener datos detallados sobre su procesamiento, y la observación para ver las funcionalidades del jefe de la dirección técnica. Se realizó una búsqueda de sistemas informáticos que pudieran emplearse para resolver las deficiencias detectadas, y al no encontrarse ninguno, se desarrolló una aplicación web con este objetivo que se ajustara a sus necesidades.

Para su desarrollo se hizo una revisión bibliográfica sobre las posibles herramientas, tecnologías y lenguajes a utilizarse según sus prestaciones, tendencias actuales y novedades, con el fin de mantener la mayor integración posible y seleccionar las adecuadas (Gutiérrez, 2008; Hernández y Greguas, 2010). Se empleó el proceso de desarrollo para guiar la metodología Extreme Programing, ya que es una metodología ágil que se basa en la realimentación continua entre el cliente y el equipo de desarrollo, comunicación fluida entre todos los participantes y simplicidad en las soluciones (Bautista, 2015; Díaz, 2009; Tinoco, Rosales y Salas, 2010). Se utilizaron los lenguajes de programación del lado del cliente HTML 5 (Gauchat, 2012) y del lado del servidor PHP en su versión 5.5.11 (Gutiérrez, 2015). Este último permite la creación de páginas web dinámicas y facilita la conexión a diferentes tipos de servidores de bases de datos. Como gestor de base de datos se hizo uso de MySQL (Casillas y Luis Alberto, 2010), ya que cuenta con un completo soporte para tablas e índices en un único archivo por base de datos, soporte transaccional, rapidez, escaso tamaño y su completa portabilidad. Se utilizó el framework Codelgniter (Álvarez, 2009) por las facilidades para la creación de sitios web complejos. Se implementa el patrón de arquitectura ModeloVista-Controlador a través del entorno de desarrollo integrado NetBeans (Corporation, 2015). Las fases que se siguieron teniendo en cuenta lo que la metodología XP establece fueron las siguientes:

1. Exploración. En ella se definió el alcance del proyecto, se especificaron las funcionalidades que se deseaban informatizar para satisfacer las necesidades del cliente mediante 30 historias de usuario, estimándose el tiempo de desarrollo de cada una, que no era superior a los tres días. Entre las definidas se encuentran: actualizar y visualizar datos de los productos, obras, almacenes, incidencias de la seguridad informática, comportamiento de la conectividad (enlace conmutado y arrendado), mantenimientos, salidas de productos de los almacenes, los datos de los usuarios con correo y conexión nacional e internacional, inventario por obras, inventario por almacenes, entre otras.

2. Plan de entrega. Se realizó una estimación detallada del tiempo a emplearse en el desarrollo de las historias de usuario y para eso se dividieron en tres iteraciones. En el cronograma de entregas se estimó que la duración de la primera iteración fuera de cuatro semanas, la segunda y la tercera tres semanas cada una. 


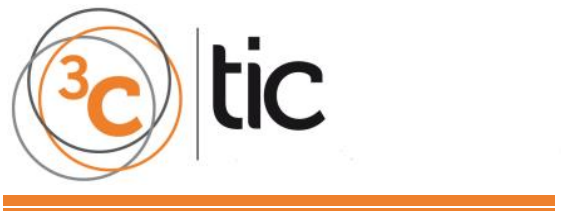

3C TIC (Edición núm. 21) Vol.6 - № 2

Junio - septiembre ' $17,28-37$

Área de Innovación y Desarrollo, S.L.

ISSN: $2254-6529$

DOI: http://dx.doi.org/10.17993/3ctic.2017.56.28-37

3. Iteraciones. Se planificó la etapa de implementación, definiéndose las tareas para desarrollar cada historia de usuario en términos de diseño y programación, se describió cada una y se estimó el tiempo de duración.

4. Producción. Se diseñó, codificó y probó la aplicación web. Tras ser creada la primera iteración, se trabajó en las nuevas.

$\checkmark$ Diseño: para lograr un sistema robusto, reutilizable y basados en las prácticas de la metodología seleccionada, se trabajó en un diseño sencillo y evolutivo. La base de datos se diseñó con 27 tablas normalizadas hasta la $3^{\text {ra }}$ Forma Normal mediante la herramienta Embarcadero ER/Studio.

$\checkmark$ Codificación: se tuvo en cuenta la reutilización de código para agilizar la implementación. Se estableció un estándar de codificación para lograr uniformidad y facilitar la comprensión del código.

$\checkmark$ Pruebas: se realizaron pruebas unitarias y de aceptación para evaluar la calidad de la aplicación web de forma pragmática y descubrir errores. Las unitarias o de caja blanca fueron desarrolladas por los programadores para verificar el código, es decir, los detalles procedimentales (la lógica del sistema). Las de aceptación también llamadas pruebas funcionales o de caja negra fueron supervisadas por el cliente, basándose en los requerimientos tomados de las historias de usuario se verificó el resultado esperado de una transacción determinada, y se llevaron a cabo sobre la interfaz. Estas pruebas permitieron encontrar errores como: funciones incorrectas, errores de interfaz, errores en estructuras de datos, errores de inicialización, errores ortográficos y la no actualización correcta de los datos. Todos los errores detectados fueron corregidos a tiempo, lográndose una aplicación web de mayor calidad.

5. Mantenimiento. Durante el desarrollo se tuvieron en cuenta las opiniones del cliente. En esta etapa se identificaron necesidades que propiciaron cambios en la aplicación web, lo que dio lugar a mantenimiento de tipo adaptativo, correctivo y perfectivo.

6. Muerte del Proyecto. Se generó la documentación final y al no requerirse más cambios en la arquitectura, al cliente no tener más historias de usuario que incluir y al estar satisfecho con el sistema, pues éste generó los beneficios esperados, se dio por terminada la aplicación web.

Una vez que finalizó la implementación de la aplicación web, esta se desplegó en la ECOAl4 y se capacitó al personal que interactúa con la misma. Además, se proporcionó un manual de usuario que contiene detalles de cómo se opera con ella para que pudiera evacuar cualquier duda sobre su manejo en el futuro. 


\section{RESULTADOS}

La aplicación web cuenta con una serie de funcionalidades que la hacen una herramienta útil para gestionar la información relacionada con productos de los almacenes de la ECOAI4. En su desarrollo se intentó satisfacer las necesidades del cliente. Su interfaz es cómoda y facilita la navegación. Es configurable, permitiendo ser implantado con gran facilidad en cualquier grupo empresarial o empresa que controle la misma información, con características similares.

En aras de garantizar la seguridad y confiabilidad de los datos que se gestionan, cuenta con una jerarquía de usuario para acceder a cualquier opción del sistema, dígase: introducir, modificar o eliminar e incluso visualizar los reportes, garantizándose así que la información que se introduzca la hagan personas debidamente autorizadas. Se tuvo en cuenta que solo accedan a los registros de la empresa a la que pertenecen y que a nivel de grupo empresarial se tenga acceso a todo. La contraseña se encriptó en la base de datos mediante el método md5. La figura 1 muestra la página de inicio.

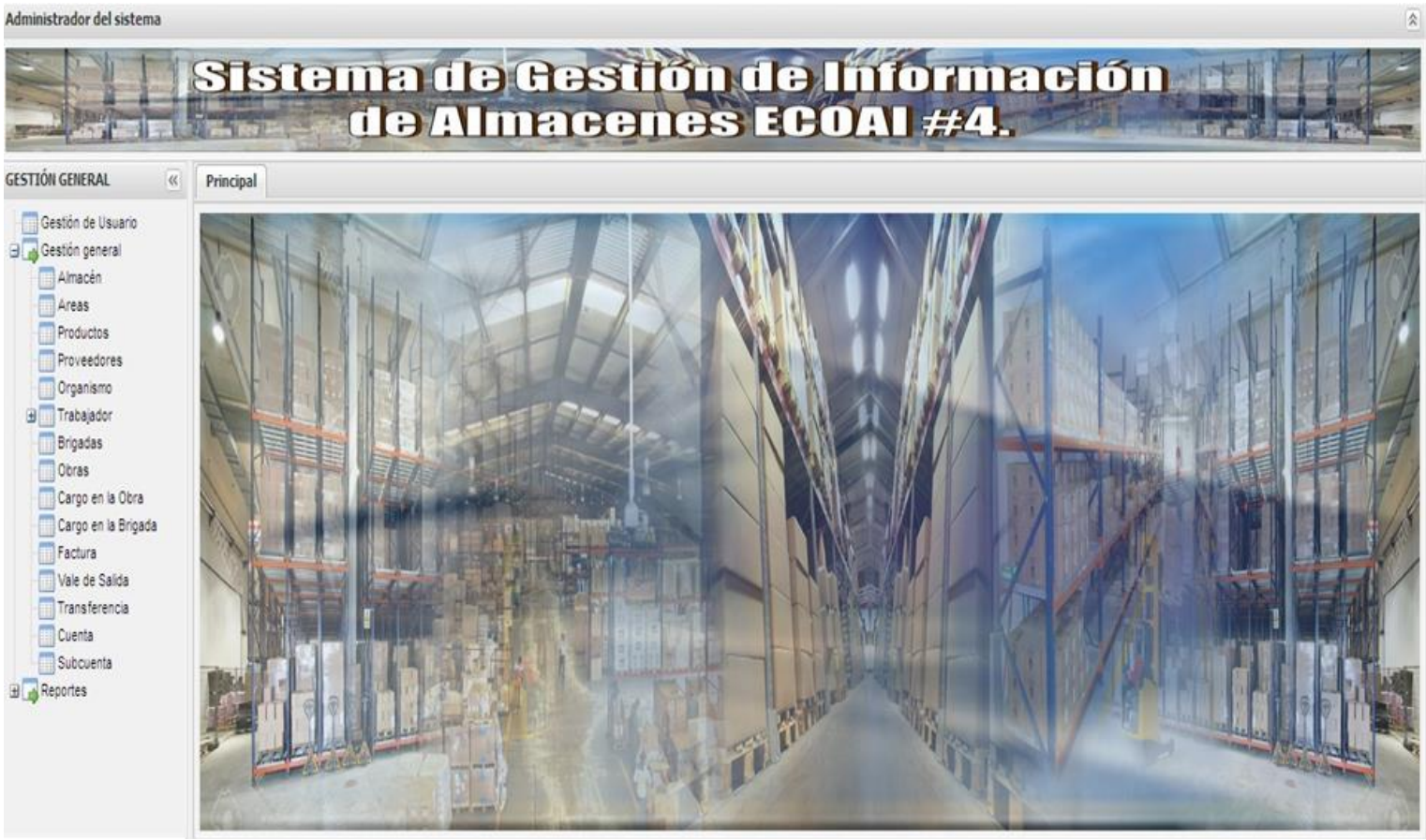

Figura 1. Página de inicio de la aplicación web.

Fuente: elaboración propia.

Entre la información que se gestiona mediante la aplicación web se encuentra la referente a los productos. En la figura 2 se observa el formulario con esta opción. Se siguió el mismo diseño para que sea cómodo al usuario, por ejemplo, el botón adicionar es para insertar un nuevo producto. 


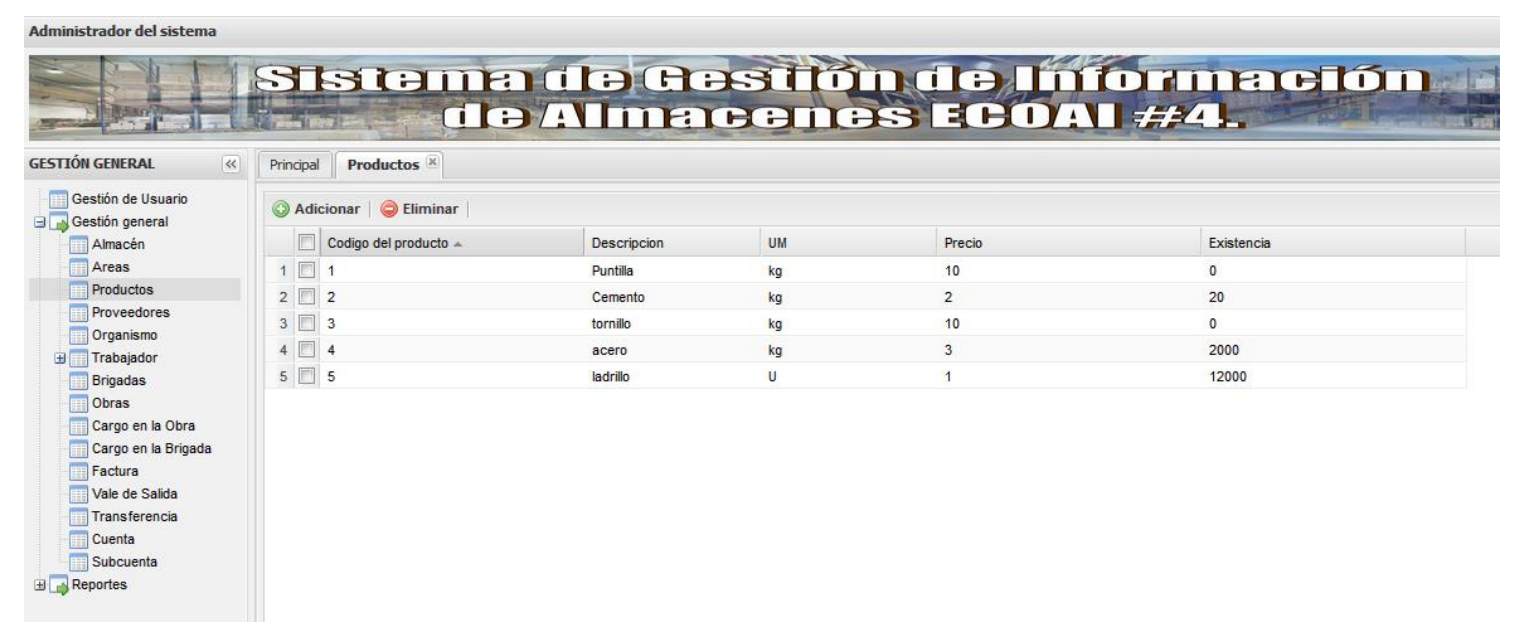

Figura 2. Gestión de los productos.

Fuente: elaboración propia.

También se pueden obtener reportes relacionados con el inventario de los productos en los almacenes, las transferencias emitidas en un intervalo de tiempo de un almacén a otro, entre otros, sustituyendo cálculos manuales y obteniendo los resultados de una forma más eficiente. Por ejemplo en la figura 3 se muestra el inventario de un almacén determinado.

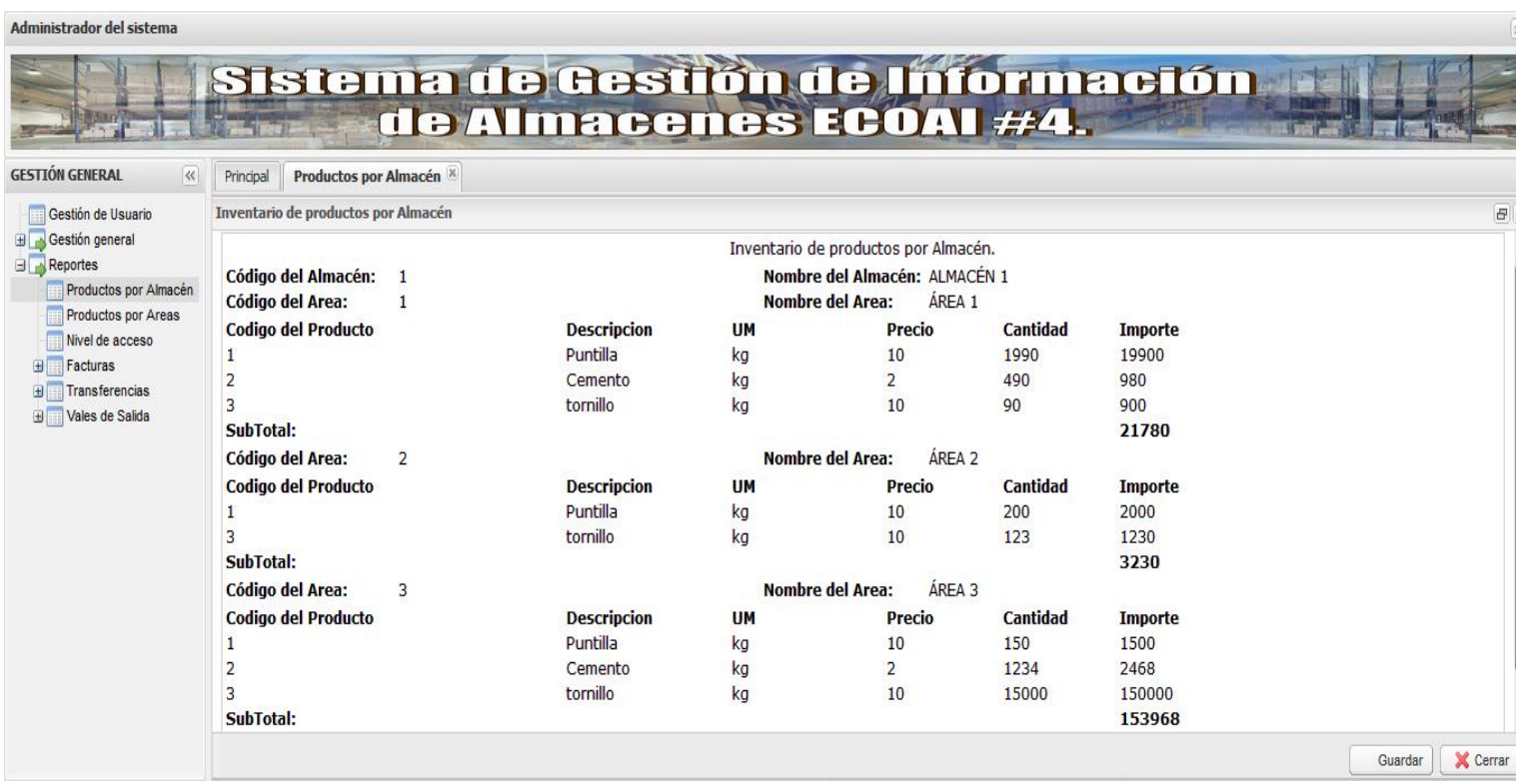

Figura 3. Reporte emitido por la aplicación web sobre inventario de un almacén determinado.

Fuente: elaboración propia.

La aplicación web brinda la facilidad de realizar salva(s) de seguridad de la base de datos cuando se estime necesario para garantizar la seguridad de la información ante la rotura del servidor o cualquier otra eventualidad. 


\section{DISCUSIÓN}

El éxito de un software se puede definir como calidad del sistema, de los datos y del servicio. La aplicación web impacta de manera positiva en los procesos y decisiones de la ECOAI \# 4, al repercutir en todos estos aspectos. Con su implantación, no es necesario mejorar el equipamiento del que se dispone pues se desarrolló en función de sus requerimientos técnicos. Las tecnologías que requiere para su funcionamiento están basadas en software libre, por lo que puede ser modificado en caso de cualquier error técnico. Se logra el ahorro de una serie de recursos como el papel al estar almacenada la información en formato digital y el combustible debido a lo distantes que están los almacenes unos de otros y en ocasiones las obras de los almacenes. Representa beneficios en cuanto al factor tiempo, los errores humanos se minimizan y el control es más sistemático.

No genera empleo ni desempleo, se facilita en gran medida la realización de las actividades laborales. Se requiere de un mínimo de esfuerzo para utilizar la aplicación web, evita el estrés y mejora la calidad de vida al poder realizarse las operaciones con mayor rapidez. Se contribuye a elevar el nivel profesional y cultural de todos los trabajadores, ya que están interactuando con la tecnología que es utilizada en la mayor parte del mundo en cuanto a medios de comunicación e informatización.

El usuario, una vez que interactuó con la aplicación web fue capaz de percibir los beneficios que esta le proporciona para evaluar su calidad se empleó el criterio de expertos mediante el método Delphi. Éste consiste en la utilización sistemática del juicio intuitivo de un grupo de expertos para obtener un consenso de opiniones informadas. Es considerado como uno de los métodos subjetivos de pronosticación más confiables (Blanco, López y Mengual, 2010; García y Suárez, 2013).

Los expertos que evaluaron la calidad de la aplicación web fueron 30. Respondieron una encuesta que se confeccionó a partir de los siguientes indicadores generales de evaluación:

1. Resulta una interfaz amigable y fácil de operar.

2. Garantiza la disponibilidad de la información actual e histórica.

3. Facilita el análisis de la información actualizada.

4. Garantiza la seguridad de la información.

5. Constituye una herramienta útil para la toma de decisiones.

6. Disminuye el tiempo de respuesta.

7. Eleva la calidad de la información.

8. Garantiza la obtención de todos los reportes que necesita.

9. Garantiza la obtención correcta de la información.

Después de realizado el procesamiento de la encuesta, se obtuvieron resultados a favor de la calidad y el impacto que tuvo la puesta en práctica en la ECOAI4 de la aplicación web para la gestión de información de los almacenes. 


\section{CONCLUSIONES}

En la presente investigación se efectuó un análisis del proceso de gestión de la información en los almacenes de la Empresa Constructora de Obras de Arquitectura e Industriales \# 4 de Granma.

Se desarrolló un sistema informático que automatiza el proceso antes mencionado facilitándole a esta institución una herramienta eficiente que reduce a cero los costos de transportación para garantizar el flujo de la información contable desde los almacenes hacia la oficina central de la empresa. La solicitud de los materiales antes de implantado el sistema dependía del transporte y de la distancia entre obras y almacenes y con el sistema es instantáneo la misma. Además, el tiempo de servir un pedido de material se redujo en más de un $80 \%$ con respecto a cómo se hacía antes de instalado el sistema. Por último, también reduce considerablemente el tiempo para efectuar los controles necesarios a la gestión de los almacenes. 


\section{REFERENCIAS BIBLIOGRÁFICAS}

Álvarez, M. A. (2009). Codelgniter es un framework PHP para la creación rápida de aplicaciones web. Recuperado el 25 de noviembre, 2015, a partir de: $<$ http://www.desarrolloweb.com/manuales/manual-codeigniter.html/>.

Bautista, J. M. (2015). EXTREME PROGRAMMING (XP). [Recuperado el de Item]. Caballero, D. (2015). Sistema de gestión de contratos Gnsis9. Recuperado el 16 de febrero, 2016, a partir de: <http://ofertas.cu/a/24895/sistema-de-gestion-de-contratos-gnsis9.html/>.

Blanco, J.E., López, A. y Mengual, S. (2010). Validación mediante método Delphi de un cuestionario para conocer las experiencias e interés hacia las actividades acuáticas con especial atención al Windsurf. ÁGORA, 12(1), 75-96.

Casillas, y. c., Luis Alberto (2010). Bases de datos en MySQL. Recuperado de: $<$ http://ocw.uoc.edu/computer-science-technology-and-multimedia/bases-dedatos/bases-de-datos/P06 M2109 02151.pdf/>.

Corporation, L. (2015). Entorno de desarrollo integrado. Recuperado el 25 de noviembre 2015, a partir de: <http://es.slideshare.net/NIRVANA27/entorno-de-desarrollointegrado?qid=ead0555a-a9c1-4e45-a1fa4673a364e30c\&v=qf1\&b=\&from search=2/>.

Díaz, J. (2009). Las metodologías ágiles como garantía de calidad del software. Revista Española de Innovación, Calidad e Ingeniería del Software (REICIS), 5(3), 40-43.

Ferrada, X.V. y Serpell, A. (2009). La gestión del conocimiento y la industria de la construcción. Revista de la Construcción, 8(1), 46-58.

García, M. y Suárez, M. (2013). El método Delphi para la consulta a expertos en la investigación científica. Revista Cubana de Salud Pública, 39(2), 253-267.

Gauchat, J. (2012). El gran libro de HTML5, CSS3 y Javascript. Barcelona España.

Gutiérrez, C. (2008). Diseño web y arquitectura de información para sitios 2.0. Cuadernos de Información, 22, 58-65.

Gutiérrez, E. G. (2015). ¿Qué es php? ¿Para qué sirve php? Un potente lenguaje de programación para crear páginas web. [Recuperado el de Item].

Hernández, R.L. y Greguas, D. (2010). Estándares de Diseño Web. Ciencias de la Información, 41(2), 69-71.

MICONS. (2014). Modelo del profesional de la especialidad Construcción Civil. Consultado: 7 de noviembre de 2014, a partir de: <http://micons.netcons.com.cu/>.

Tinoco, O., Rosales, P. P. y Salas, J. (2010). Criterios de selección de metodologías de desarrollo de software. Industrial Data, 13(2), 70-74. 